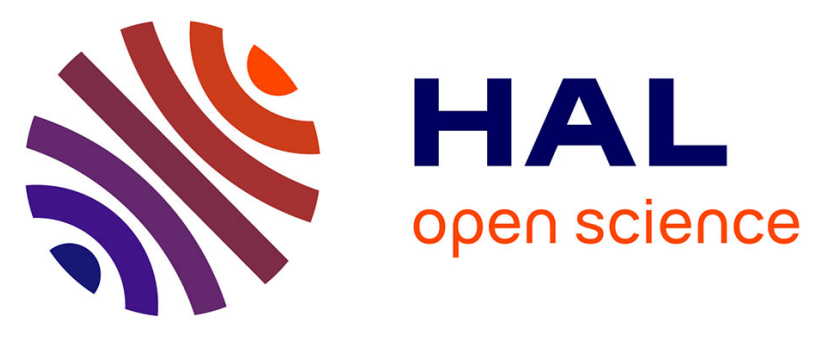

\title{
Les fibres alimentaires limitent le stockage de lipides hépatiques en situation de surnutrition : quels mécanismes et quels médiateurs?
}

Ahmed Ben Mohamed, Didier Remond, Guy Della Valle, Hubert Chiron, Benoit Cohade, Jérémie David, Dominique Dardevet, Joel Dore, Isabelle Savary-Auzeloux

\section{To cite this version:}

Ahmed Ben Mohamed, Didier Remond, Guy Della Valle, Hubert Chiron, Benoit Cohade, et al.. Les fibres alimentaires limitent le stockage de lipides hépatiques en situation de surnutrition: quels mécanismes et quels médiateurs?. JFN 2017, Journées Francophones de Nutrition, Dec 2017, Nantes, France. 2017. hal-02738304

\section{HAL Id: hal-02738304 \\ https://hal.inrae.fr/hal-02738304}

Submitted on 2 Jun 2020

HAL is a multi-disciplinary open access archive for the deposit and dissemination of scientific research documents, whether they are published or not. The documents may come from teaching and research institutions in France or abroad, or from public or private research centers.
L'archive ouverte pluridisciplinaire HAL, est destinée au dépôt et à la diffusion de documents scientifiques de niveau recherche, publiés ou non, émanant des établissements d'enseignement et de recherche français ou étrangers, des laboratoires publics ou privés.

\section{(ㅇ)(1) $\$$}

Distributed under a Creative Commons Attribution - NonCommercial - NoDerivatives| 4.0 


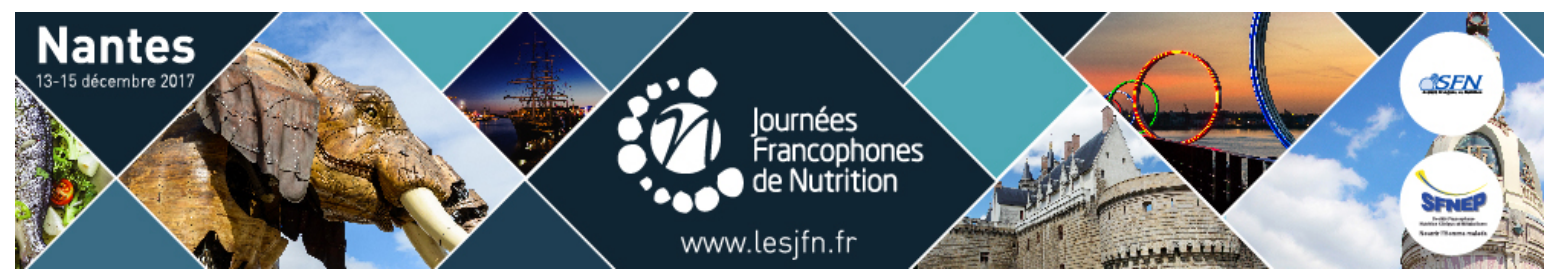

Métabolisme des macro- et micronutriments

JFN2017/1101

\section{Les fibres alimentaires limitent le stockage de lipides hépatiques en situation de surnutrition : quels mécanismes et quels médiateurs? \\ Ahmed Ben Mohamed ${ }^{*}$, Didier Rémond ${ }^{1}$, Guy Della-valle ${ }^{2}$, Chiron Hubert ${ }^{2}$, Cohade Benoit ${ }^{1}$, Jeremie David ${ }^{1}$, Dominique Dardevet $^{1}$, Joel Dore ${ }^{3}$, Sergio Polakof ${ }^{1}$, Isabelle Savary-Auzeloux ${ }^{1}$ \\ ${ }^{1} \mathrm{UNH}$, INRA, Clermont Fd, ${ }^{2} \mathrm{BIA}$, INRA, Nantes, ${ }^{3}$ Metagenopolis, INRA, J. en Josas, France}

Discipline: Expérimental/mécanismes cellulaires et moléculaires

Présentation préférée: Communication orale

Introduction et but de l'étude: Alors que l'obésité peut s'expliquer par une surnutrition et l'ingestion de régimes riches en graisses et sucres, l'ingestion de fibres alimentaires est connue pour favoriser la perte de poids ${ }^{1}$. Notre étude vise à déterminer si, en situation d'apport alimentaire supérieur aux besoins (alimentation de type occidentale générant de l'obésité), les fibres alimentaires peuvent limiter l'apparition des dérégulations métaboliques via notamment une réorganisation de l'utilisation des nutriments dans l'aire splanchnique (ASP : foie + tube digestif (TD)).

Matériel et méthodes: 14 Miniporcs sont multicathétérisés en artère, veine porte et veine sus-hépatique pour mesurer les flux nets de nutriments au travers de l'ASP. Les animaux ont été nourris 60 jours avec un régime en surnutrition $(14911 \mathrm{~kJ} / \mathrm{j}$ EM) supplémenté en pain enrichi $(\mathrm{F})(+2291 \mathrm{kj} / \mathrm{j} \mathrm{EM})$ ou non $(\mathrm{T})(+2438 \mathrm{kj} / \mathrm{j}$ EM) en fibres (inuline, pectine, amidon résistant). Des prélèvements sanguins ont été réalisés à jeun après 1,14 et 60 jours de régime $\mathrm{T}$ ou $\mathrm{F}$, ainsi que des prélèvements tissulaires à l'euthanasie. Des analyses plasmatiques (AGCC, glucose, lactate, acides aminés) et hépatiques (histologie : red oil) ont été effectuées. Les flux nets des nutriments par l'ASP ont été calculés par différence artério-veineuse. Les effets régimes/cinétiques ont été évaluées par ANOVA à mesures répétées, signification $\mathrm{P}<0,05$.

Résultats et Analyse statistique : A J60, le contenu lipidique dans le foie est inferieur chez F vs T $(-33,6 \% ; \mathrm{P}<0,01)$. Ceci est associé à une forte émission nette à jeun de glucose, lactate et acides aminés par le foie (F vs T : $+79 \mathrm{~J} 60,+108 \% \mathrm{~J} 60 ;+118 \%$ $\mathrm{J} 14 ; \mathrm{P}<0,05)$. Ainsi, l'énergie est moins stockée au niveau hépatique et redistribuée vers les tissus périphériques chez $\mathrm{F}$. Ceci est combiné à une augmentation des niveaux artériels d'alanine chez $\mathbf{F}$ vs $\mathrm{T}(+24 \%, \mathrm{~J} 60, \mathrm{P}<0.05)$ ce qui suggère une utilisation du glucose vers la synthèse d'alanine dans le muscle et une réorientation des voies d'utilisation/synthèse du glucose dans le foie et le muscle chez F.

Parmi les facteurs pouvant expliquer les changements dans l'utilisation des nutriments énergétiques entre $\mathrm{T}$ et $\mathrm{F}$, on constate une émission des AGCC par le TD supérieure chez $\mathbf{F}$ vs T uniquement à J14, liée à la dégradation des fibres solubles dans le colon. Or, les AGCC sont connus pour réguler les voies du métabolisme énergétique via une action sur GPR41-432. Néanmoins, l'effet des fibres perdurant à J60, des médiateurs relai ou des régulations à long terme doivent s'être mis en place.

Conclusion: La supplémentation en fibres alimentaires limite le stockage des lipides dans le foie en situation de surnutrition. Cet effet pourrait être médié dans les phases précoces du développement des dérégulations métaboliques par les AGCC via une altération de la répartition des nutriments énergétiques entre le foie et les tissus périphériques. Pour analyser cette hypothèse, des expressions de gènes, des analyses de protéomique et des activités enzymatiques du métabolisme énergético-azoté (foie, muscle, tissu adipeux) sont en cours.

Références: ${ }^{1}$ Obépi/étude NUTRINET-SANTE, 2012

${ }^{2}$ Butyrate Improves Insulin Sensitivity. Zhanguo Gao, Diabetes 2009

Conflits d'intérêts: Aucun conflit à déclarer 